\title{
COUPLING 3D GEOMECHANICS TO CLASSICAL PETROLEUM SYSTEM SIMULATION
}

\section{A. BRÜCH ${ }^{1 *}$, D. COLOMBO ${ }^{1}$, J. FREY ${ }^{1}$, J. BERTHELON ${ }^{1}$, M. C. CACAS-STENTZ1, T. CORNU ${ }^{2}$}

\author{
${ }^{1}$ IFP Energies nouvelles, 1 et 4 avenue de Bois-Préau, 92852 Rueil-Malmaison, France \\ 2 TOTAL S.A., Avenue Larribau, 64018 Pau, France \\ * Corresponding author. E-mail address: andre.reinert-bruch@ifpen.fr
}

Key words: Sedimentary Basin, Tectonics, Mechanical Compaction, Coupled Geomechanics, Poro-Elastoplasticity, Finite Element Method.

\begin{abstract}
Geomechanical models of classical petroleum system simulators are limited to 1D phenomenological laws relating porosity to vertical effective stress. In order to overcome this limitation, a 3D poromechanical model is integrated in the sedimentary basin simulation by applying an iterative coupling scheme between a conventional basin code and a mechanical finite element code. This paper presents the porous material constitutive law specifically devised to deal with basin modeling, together with essential aspects of the mechanical code implementation and explicit coupling workflow. The numerical procedure is first verified according to a semi-analytical solution and then compared to an implicit academic code. Finally, a 3D synthetic case demonstrates the importance of incorporating 3D geomechanics to basin simulation. The results show that tectonic compression may significantly contribute to overpressure development and natural fracturing of seal rocks, contrary to the standard procedures of petroleum system simulation which are unable to capture such effect.
\end{abstract}

\section{INTRODUCTION}

Relying upon available data and postulated geological scenarios, Petroleum System Modeling (PSM) is used by the petroleum exploration industry to locate and estimate the amount of oil and gas in hydrocarbon reservoirs [1]. It can also provide important information for well development and production teams about fluid overpressure, cap rock integrity and fault stability. A comprehensive modeling of a sedimentary basin thus requires the integration of multiple coupled phenomena such as sediment compaction, fluid flow and heat transport in order to predict rock porosity and permeability, hydrocarbon generation and migration path, as well as the identification of critically stressed zones.

However, geomechanical models of classical petroleum system simulators are restricted to vertical compaction based on phenomenological laws relating porosity to vertical effective stress. To overcome this limitation and to account for a 3D poromechanical framework, an iterative coupling scheme between a sedimentary basin code and a mechanical finite element code is adopted. This procedure allows to capture the impact of tectonics-induced deformation that may strongly affect the poromechanical state of the basin. The constitutive law used in the mechanical code has been specifically devised to deal with mechanical compaction of 
sedimentary basins in the context of finite poroplasticity. The workflow performance is improved through development of a tangent stiffness operator and HPC implementation of the coupled code, allowing to carry out simulations on large 3D models in a reasonable time.

The decision to integrate an explicit coupling instead of developing an implicit formulation on the PSM code stems to the fact that it provides more flexibility and efficiency in code management [2]. In this circumstance, two important features must be thoroughly addressed regarding the numerical modeling of sedimentary basins: a) the staggered solution between both codes and the convergence of the poro-mechanical problem, and b) the geometrical consistency between the backward and forward basin history. This work deals only with the former aspect, presenting a verification process of the proposed coupling procedure. Additional aspects regarding the backward/forward consistency as well as other theoretical components of the PSM code are not in the scope of this paper.

The constitutive model and main computational aspects are described in sections 2 and 3 , respectively. Section 4 presents the workflow verification process as follows: first, the mechanical code implementation is verified by means of a semi-analytical solution in the simplified case of one-dimensional compaction in drained conditions. Then, the effectiveness of the iterative coupling scheme is proved by comparison with results obtained within a fully implicit hydro-mechanical code for both gravitational compaction and tectonic loading situations. Finally, section 5 illustrates a 3D synthetic case study, highlighting the relevance of incorporating 3D geomechanics into sedimentary basin modeling.
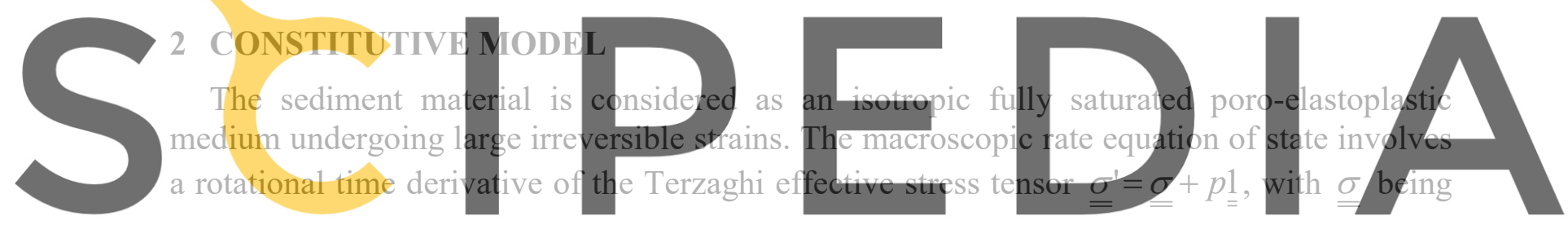

the Cauchy total stress tensor, $p$ the pore- pressure and 1 the second-order identity tensor [3]:

Register for free at https//www.scipedia.com to download the version without the watermark $\frac{D_{J} \underline{\underline{\sigma}}}{D t}=\underline{\underline{\sigma}}^{\prime}+\dot{\sigma}^{\prime} \cdot \underline{\underline{\Omega}}-\underline{\underline{\Omega}} \cdot \underline{\underline{\dot{\sigma}^{\prime}}}=C:\left(\underline{\underline{d}}-\underline{\underline{d^{p}}}\right)$

where $\underline{\underline{d}}$ and $\underline{\underline{d}}^{p}$ are respectively the total and plastic part of the strain rate tensor and $\underline{\underline{\Omega}}$ is the spin rate tensor. The fourth-order tensor $C$ is the material drained elastic stiffness moduli which by virtue of isotropy can be written as a function of the bulk $K$ and shear $\mu$ moduli as follows, with 1 referring to the fourth-order identity tensor:

$$
\underset{\sim}{C}=(K-2 \mu / 3) \underline{\underline{\sim}} \underset{\underline{\underline{1}}}{\otimes}+2 \mu \underset{\sim}{1}
$$

From mechanical viewpoint, porosity is a key parameter to evaluate stiffness increase and hardening evolution. Under the assumption of infinitesimal elastic strains for the porous material skeleton and plastic incompressibility of its solid phase, the Eulerian porosity $\varphi$ can be computed as a function of the Jacobian of plastic transformation $J^{p}$ : 


$$
\varphi=1-\frac{1-\varphi_{0}}{J^{p}}
$$

The evolution of the elastic properties are quantified according to the upper bounds of Hashin-Shtrikman, where $k^{s}$ and $\mu^{s}$ are the bulk and shear moduli of the solid phase [4]:

$$
K(\varphi)=\frac{4 k^{s} \mu^{s}(1-\varphi)}{3 k^{s} \varphi+4 \mu^{s}} \text { and } \mu(\varphi)=\frac{\mu^{s}(1-\varphi)\left(9 k^{s}+8 \mu^{s}\right)}{k^{s}(9+6 \varphi)+\mu^{s}(8+12 \varphi)}
$$

The plastic model represents purely mechanical compaction of the sediment material. The yield surface is taken from the modified Cam-Clay model, written as a function of the deviatoric stress tensor $\underline{\underline{s}}=\underline{\underline{\sigma}}-\operatorname{tr} \underline{\underline{\sigma}} / 3 \underline{\underline{1}}$ and the mean effective stress $p^{\prime}=\operatorname{tr} \underline{\underline{\sigma^{\prime}}} / 3[5]$ :

$$
f=\frac{3}{2}=\stackrel{S}{=}+M_{c s}^{2}\left(p^{\prime}-p_{t}\right)\left(p^{\prime}+p_{c}\right)
$$

where $p_{c}$ is the consolidation pressure and represents the hardening parameter of the model, $p_{t}$ is the tensile intercept of the yield surface with the hydrostatic axis and $M_{c s}$ is the slope of the critical state line. An associated flow rule is adopted for the plastic strain rate:
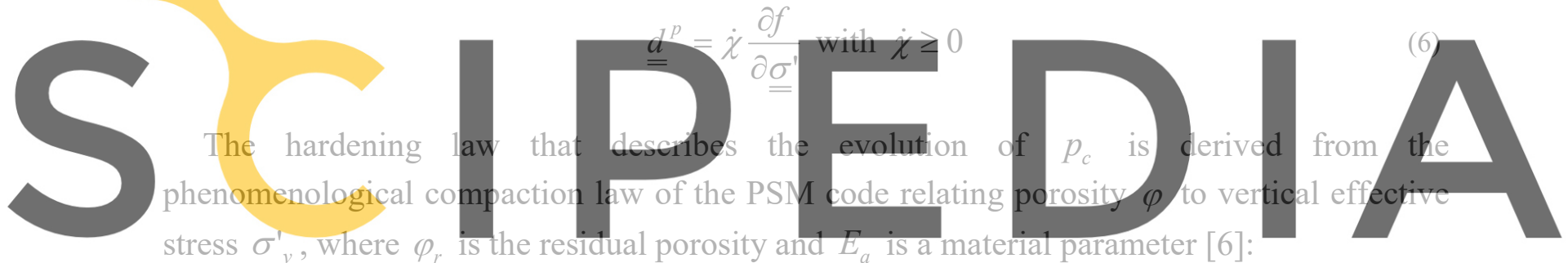

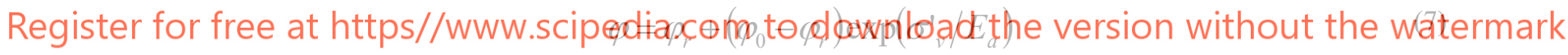

By parameters identification, the hardening law for the consolidation pressure writes:

$$
p_{c}=C \ln \left(\frac{\varphi_{0}-\varphi_{r}}{\varphi-\varphi_{r}}\right)
$$

where $C$ is a calibration coefficient. It can be verified that $p_{c} \rightarrow+\infty$ when $J^{p} \rightarrow \frac{1-\varphi_{0}}{1-\varphi_{r}}$, i.e. when porosity reaches its residual value, excluding the possibility to have negative porosities.

\section{COMPUTATIONAL ASPECTS}

The solution of the mechanical problem is achieved by solving at each instant of time a specific boundary value problem formulated on the geometrical domain $\Omega$ of the considered material system. It is defined by the set of the field, constitutive and complementary equations, and completed by the initial values of all field variables together with the boundary conditions that should be prescribed on the boundary $\partial \Omega$ of $\Omega$. 


\subsection{Finite element discretization}

Disregarding inertial effects, the momentum balance equation reads:

$$
\operatorname{div} \underline{\underline{\sigma}}+\rho \underline{g}=0
$$

with $\rho=(1-\varphi) \rho^{s}+\varphi \rho^{f}$, where $\rho$ is the density of the porous material, $\rho^{s}$ and $\rho^{f}$ are the mass densities of the solid and fluid phases, and $\underline{g}$ is the acceleration of gravity.

The discretized form of the mechanical problem is obtained from weak formulation of the equilibrium equation at time $t^{\prime}=t+\Delta t$ (unknown configuration) employing piecewise linear functions for the displacement $\underline{U}$. The updated Lagrangian scheme [7] is used to analyze the evolution of the basin between its configuration at time $t$ (updated reference configuration) and that at time $t^{\prime}$. The unknown variables are then updated at each time step $\Delta t$.

Denoting by ${ }^{t} \underline{x}$ the coordinate vector of a sediment particle at time $t$, the boundary value problem is reformulated in terms of displacement $U$ of the particle between $t$ and $t^{\prime}$ :

$$
\underline{U}={ }^{t^{\prime}} \underline{x}-{ }^{t} \underline{x}
$$

The pore-pressure field obtained from the PSM code is discretized in time according to the mechanical scheme. The pressure variation at points similar within the porous material reads:
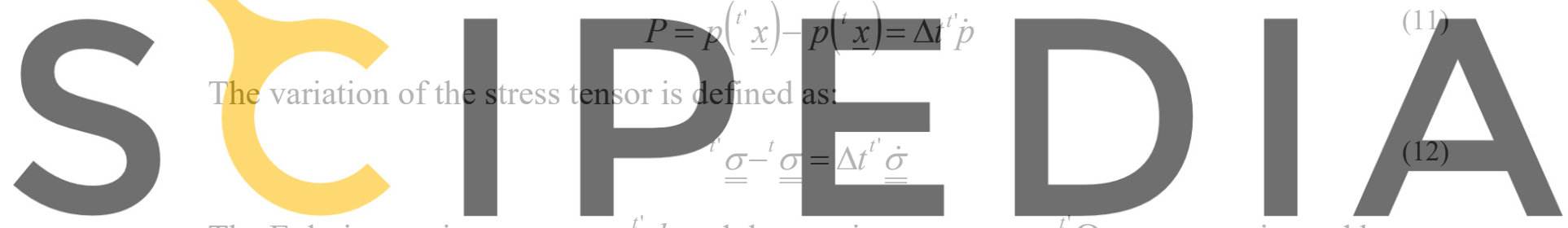

The Eulerian strain rate tensor ${ }^{t} d$ and the rotation rate tensor ${ }^{t} \underline{\underline{ }}$ are approximated by:

Register for free at https//www.scipedia.com to download the version without the watermark

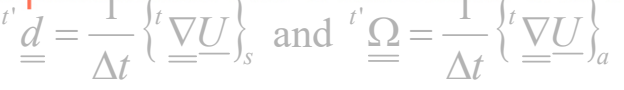

The hypothesis of infinitesimal transformation is adopted by respecting the condition $\left|{ }^{t} \underline{\underline{\nabla}} \underline{U}\right|<<1$ for each converged time step. As a consequence, the geometrical nonlinearities are disregarded in the numerical procedure. The resulting expression of Eq. (1) for time $t^{\prime}$ is:

$$
\underline{\underline{\sigma}}={ }^{t} \underline{\underline{\sigma}}+{ }^{t^{\prime}} C^{e p}:\{\underline{\underline{\nabla}} \underline{\underline{U}}\}_{s}-P \underline{\underline{1}}
$$

where $C^{e p}$ accounts for the consistent tangent stiffness operator [8]:

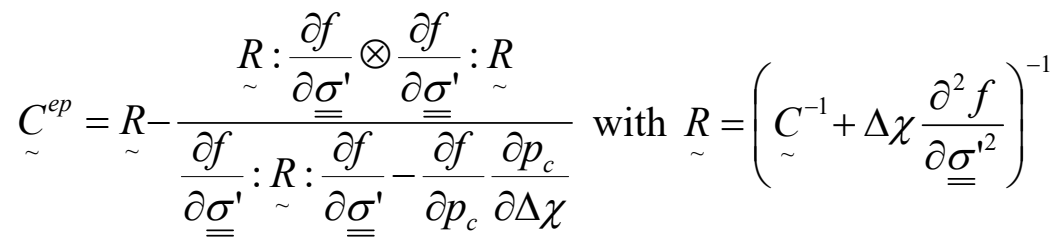


The mechanical problem is then solved for time $t^{\prime}$ by substituting Eq. (14) in the weak form of the equilibrium equation, considering ${ }^{t^{\prime}} C \approx^{t} C,{ }^{t^{\prime}} \rho \approx^{t} \rho, \partial \Omega_{t^{\prime}} \approx \partial \Omega_{t}$ and $\Omega_{t^{\prime}} \approx \Omega_{t}$. These approximations imply that the only nonlinear term of the numerical formulation concerns the fourth order tensor ${ }^{t^{\prime}} C^{e p}$ due to the plastic evolution of the porous material.

\subsection{Explicit coupling strategy}

The sedimentary basin history is defined in the PSM code by a sequence of geological events. The iterative coupling strategy considers these events as the time periods for which the staggered solution between the PSM and mechanical codes should be repeated until convergence is reached. During these periods, each code has its own time discretization and independent porosity evaluation. Therefore, the convergence criterion used for the iterative coupling is based on the porosity difference between the two codes.

For a given geological event, the first calculation is performed by the PSM code. The porepressure variation obtained at the end of the geological event is transmitted to the mechanical code where it is used in Eq. (14) in order to calculate a three-dimensional displacement field. The porosity distribution resulting from the 3D geomechanics solution is then compared to that obtained from the simplified 1D compaction law of the basin simulator. If the porosity difference is lower than a chosen tolerance, the numerical simulation proceeds to the next event. Otherwise, a porosity correction method is applied to correct the inconsistency between the porosity computed from both codes. This type of predictor-corrector approach is well known for coupling geo

Keeping in mind tha

effective stress $\sigma_{v}^{\prime}=\sigma_{v}+p$

code to integrate the eft

ith iteration of the staggered procedure, the vertical stress considered in the PSM code reads:

Register for free at https//www.scipedia.com to download the version without the watermark

Depending on the permeability distribution of the basin, the corrective term $\Delta \sigma_{v}$ will affect the porosity and/or pore-pressure fields in the PSM code. The pressure variation is then injected again in the mechanical code. The scheme is repeated up to porosity convergence.

\section{WORKFLOW VERIFICATION}

In addition to the finite element discretization of the mechanical problem, one specificity of dealing with numerical simulation of sedimentary basins is the fact that an open material system is considered [10]. In that regard, specific numerical procedures are adopted to model sediments deposition and erosion processes, so that the basin is represented as a fictitious closed system. The evolution in time of the latter must correspond to that of the real system.

\subsection{Mechanical code implementation}

The ability of the mechanical code to deal with sedimentary basin modeling is verified in this section by means of a semi-analytical solution in the case of one-dimensional compaction 
in drained and isothermal conditions, disregarding pressure and temperature effects. The problem briefly described in this section is based on existing analytical solutions [11].

The basin is modeled as an infinite layer, perpendicular to the $\underline{e}_{z}$ direction, so that all quantities of the problem depend only on time $t$ and vertical coordinate $z$. The origin of the coordinate system coincides with the top of the basin. The bottom, considered rigid, is located at the plane $z=-H(t)$, where $H$ is the basin thickness, function of the rate of sediment mass supply per unit area $\dot{M}_{d}$ and the particle velocity at the top of the basin, $V_{z, t o p}=V_{z}(z=0)$ :

$$
\dot{H}=\frac{\dot{M}_{d}}{\rho_{0}}+V_{z, t o p}
$$

In addition to the equilibrium Eq. (9), the mass balance equation must be considered:

$$
\frac{\partial \rho}{\partial t}+\operatorname{div}(\rho \underline{V})=0
$$

The constitutive model is the same of section 2 with the exception for the plastic yield surface for which a simplified cap model is considered, where $N$ is a material constant:

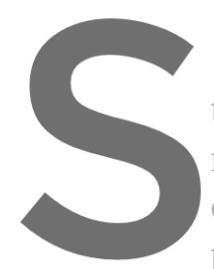

$$
f=\sqrt{\frac{1}{2} \stackrel{S}{=}: \underline{S}}-N\left(p^{\prime}+p_{c}\right)
$$

An Eulerian quantity time $t$ with respect to th monotonic sedimentatio conveniently be regarded as partial differential equat
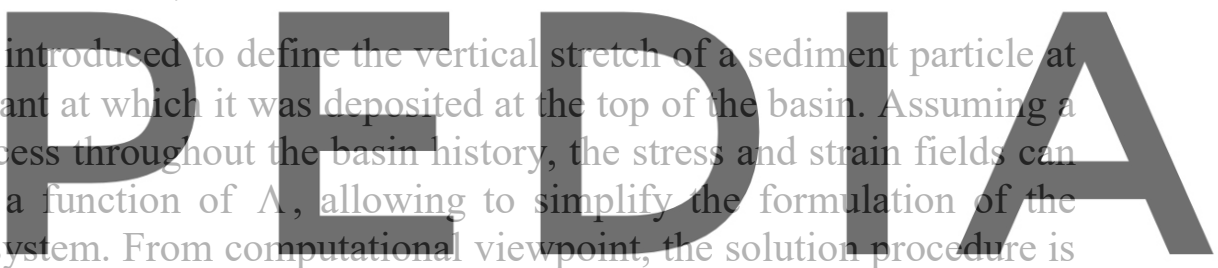

performed incrementally by discretizing the governing equations in time. Starting from a

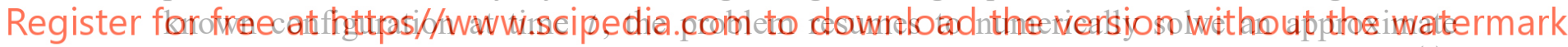
system of ordinary differential equations for each time step $\Delta t$, with a known thickness $H(t)$.

The procedure allows thus for the determination of the new basin configuration at time $t+\Delta t$. In particular, the basin thickness is updated using equation (17) in discretized form:

$$
H(t+\Delta t)=H(t)+\Delta H \text { with } \Delta H=\Delta t\left(\frac{\dot{M}_{d}(t)}{\rho_{0}}+V_{z, t o p}(t)\right)
$$

The resulting system of equations for the field, constitutive and complementary equations is given in Eq. (21) together with the boundary conditions. A finite difference technique is used to solve the system at each time step. The rate of sediment material supply is considered as constant equal to $\dot{M}_{d}=4.28 \times 10^{-9} \mathrm{~kg} / \mathrm{s}$ per unit area (or $\dot{M}_{d}=135 \mathrm{ton} / \mathrm{km}^{2} /$ year) for a total time of $T_{\text {sed }}=60 \mathrm{My}$. Porous material initial properties are: density $\rho_{0}=1350 \mathrm{~kg} / \mathrm{m}^{3}$, porosity $\varphi_{0}=50 \%$, Young modulus $E_{0}=5000 \mathrm{MPa}$ and Poisson's ratio $v_{0}=0.25$. For the hardening law (8), $C=20 \mathrm{MPa}$ and $\varphi_{r}=1 \%$, and for the yield function (19), $N=0.866$. The adopted values for $\dot{M}_{d}$ and $\rho_{0}$ correspond to a sedimentation rate of $100 \mathrm{~m} / \mathrm{My}$. 


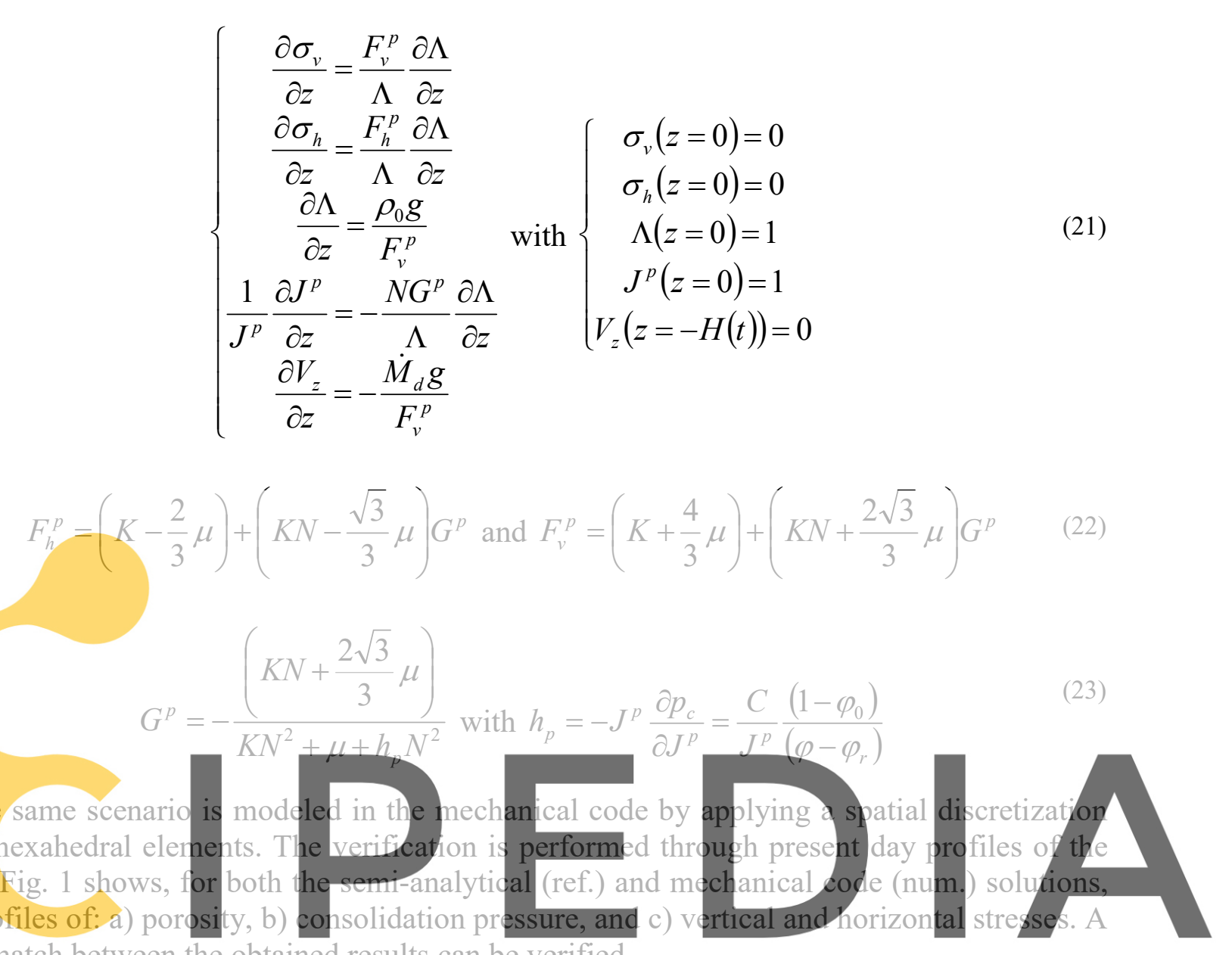

good match between the obtained results can be verified.

Register for free at https//www.scjpedia.com to download the version without the watermark
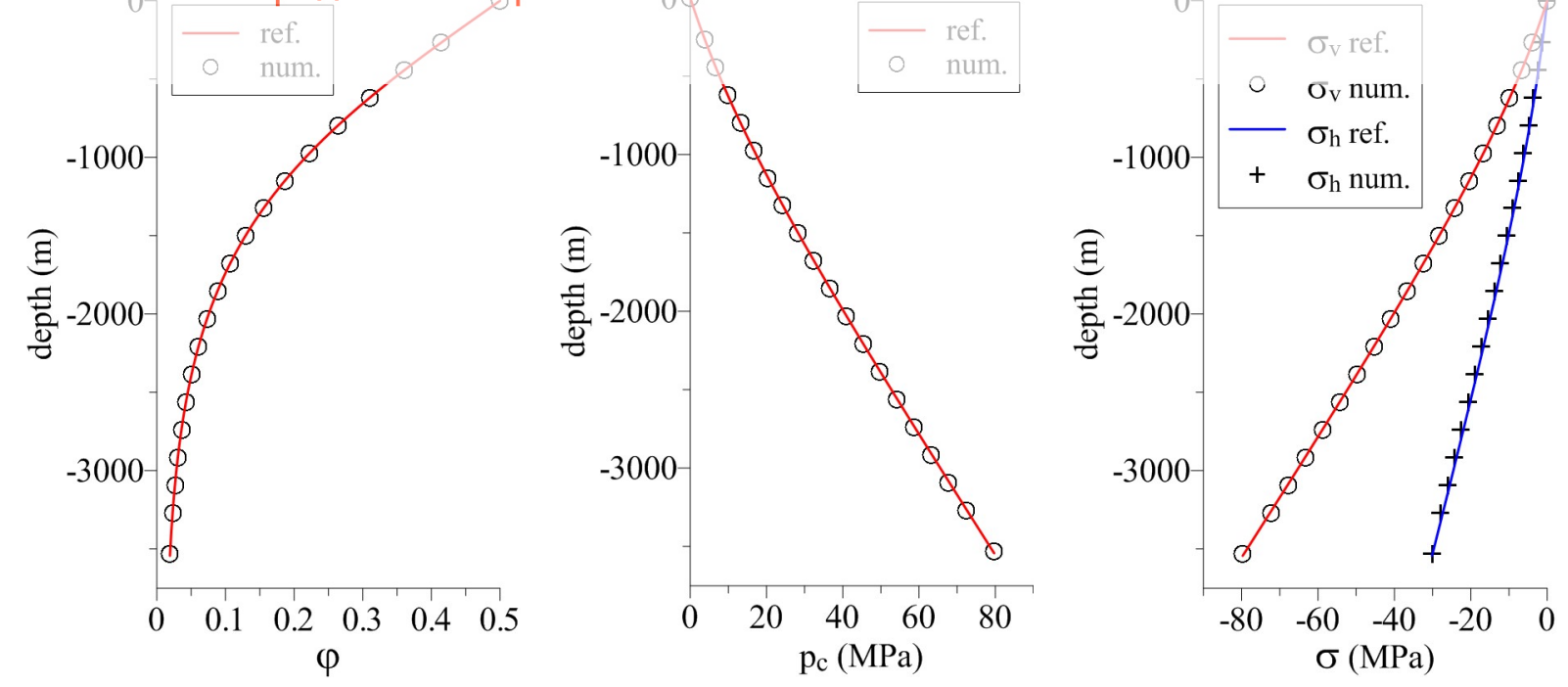

Figure 1: Basin profiles at present day for semi-analytical (ref.) and mechanical code (num.) solutions 


\subsection{Poromechanical coupling: gravitational compaction}

The objective now is to illustrate the effectiveness of the coupling scheme proposed in section 3 applied to the constitutive model of section 2 . The results are compared to a fully implicit solution from an academic code specifically devised for dealing with forward basin modeling [12]. An additional simulation is also carried out using the PSM code alone.

A synthetic case of a deposition sequence of high permeability sandstones and low permeability shales under gravitational compaction is investigated. The basin is modeled as a vertical column under oedometric conditions with impermeability conditions at the bottom and side surfaces, while the top surface is set to $p=0$. Temperature effects have not been considered to keep the problem restricted to the poro-mechanical coupling verification.

The geological sequences take place as follows: 1) a sandstone compartment is deposited in the first $20 \mathrm{My}$ at constant rate of $144 \mathrm{~m} / \mathrm{My}, 2$ ) in the sequel, shale seal rocks are deposited during $30 \mathrm{My}$ at $53 \mathrm{~m} / \mathrm{My}, 3$ ) finally, a sandstone overburden is deposited in the last $40 \mathrm{My}$ at $58.5 \mathrm{~m} / \mathrm{My}$. Both compartment and overburden sandstones have the same poromechanical parameters. Mechanical data are given in Table 1. The corresponding parameter $E_{a}$ from the PSM compaction law (7) is also given for comparison purposes.

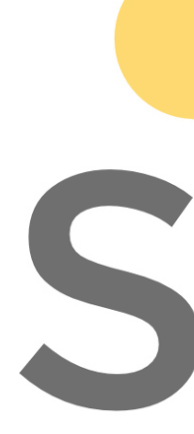

Table 1: Mechanical data for sandstone and shale materials

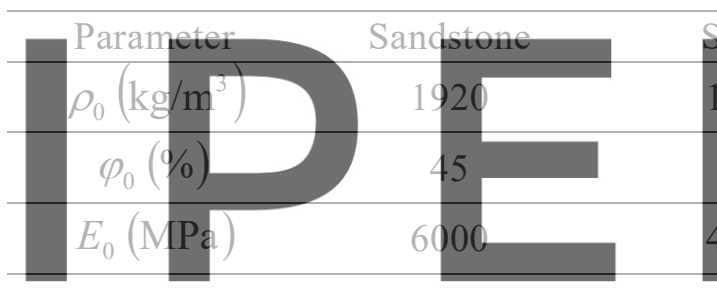

$v_{0} \quad 0.25$

0.25
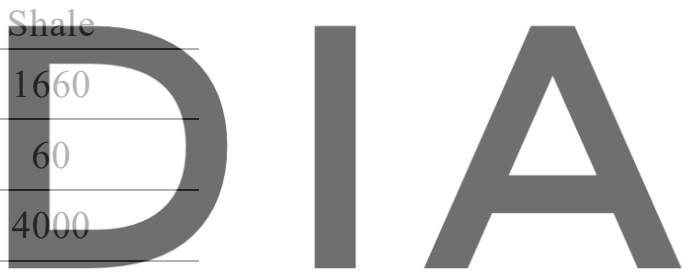

Register for free at https//wwv.\$cip@dia.com to5ofownload theoversion without the watermark

\begin{tabular}{ccc}
\hline$\varphi_{r}(\%)$ & 1 & 4 \\
\hline$M_{c s}$ & 1.2 & 1.2 \\
\hline$p_{t}(\mathrm{MPa})$ & 0.25 & 0.25 \\
\hline$E_{a}(\mathrm{MPa})$ & 40 & 12 \\
\hline
\end{tabular}

Present day profiles of the basin are illustrated in Fig. 2 for: a) porosity, b) pressure and overpressure, and c) vertical and horizontal effective stresses. The results concern the explicit coupling (EXP), the implicit academic code (IMP) and the PSM code. Two major conclusions can be made: 1) the constitutive model of section 2 provides the same porosity distribution of the PSM code when submitted to oedometric conditions, 2) the iterative coupling strategy of section 3 seems reliable as the results match those of the implicit coupling. 

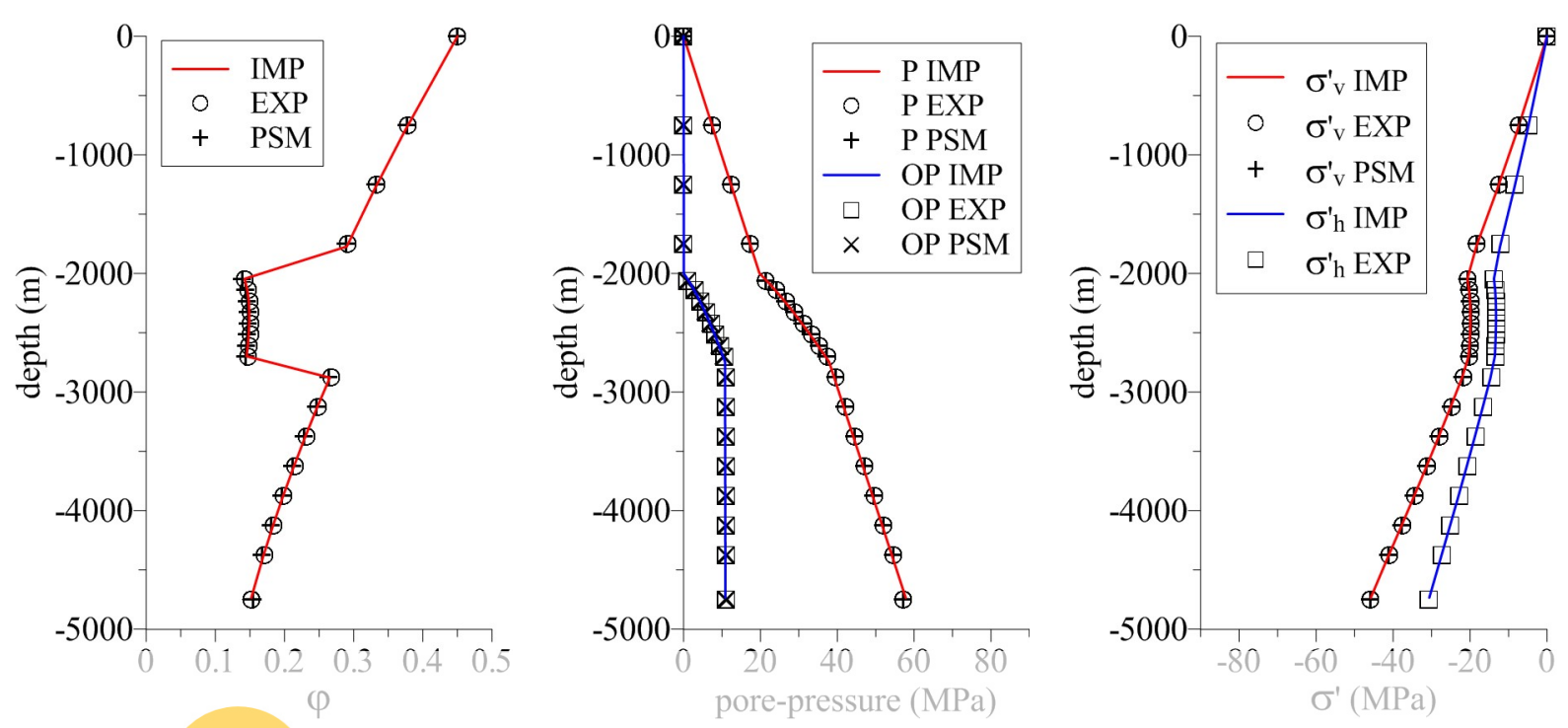

Figure 2: Basin profiles at present day for gravitational compaction

(IMP = implicit academic code; EXP = explicit coupling workflow; PSM = conventional basin code)

\subsection{Poromechanical coupling: tectonic loading}

In order to continue with the verification process of the coupling workflow while proving the relevance of incorporating 3D geomechanics into sedimentary basin modeling, deals with lateral deformations induced by sequences described in the previous case, the basi during 2 My at constant rate. The restlting basin profiles are match between the explicit and implicit methods is observed.
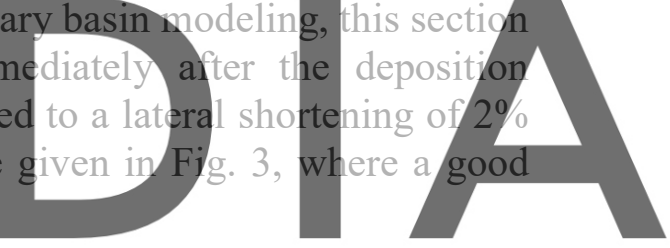

Register for free at https//www.scipedia.com to download the version witho untathe waitermark
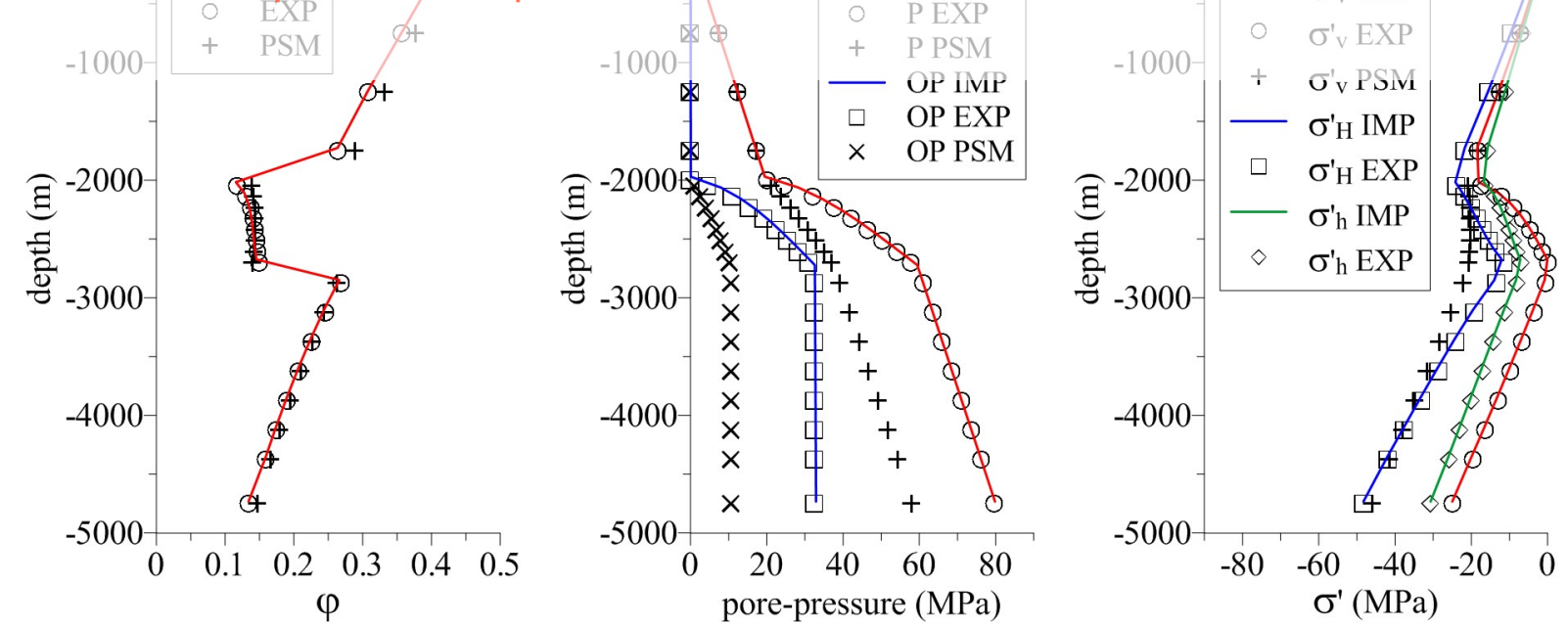

Figure 3: Basin profiles at present day for tectonic loading (IMP = implicit academic code; EXP = explicit coupling workflow; PSM = conventional basin code) 
Results from the PSM code, which accounts for gravitational compaction only, are also reported for comparison. The discrepancy of these results with respect to the others can be readily seen. The simplified one-dimensional geomechanics of the conventional basin simulator and the consequent lack of capacity to take into account lateral deformations has led the basin to a completely different poromechanical state.

\section{ILLUSTRATIVE 3D CASE}

A synthetic case is chosen to illustrate the explicit coupling workflow in a threedimensional framework. The geological scenario is based on those of the previous sections: the deposition history is the same of section 4.2 followed by the tectonic shortening of section 4.3. The main difference is that the tectonic phase leads to the initiation of a buckle fold in the center of the basin, where the resulting uplift is partially eroded. In addition, the geothermal evolution is taken into account by prescribing a constant upward heat flow $q^{t}=50 \mathrm{~mW} / \mathrm{m}^{2}$ at the bottom, and a constant temperature $T=20^{\circ} \mathrm{C}$ at the top of the basin, while keeping the lateral surfaces thermally insulated. The geometry is defined in the horizontal plane by a square with $50 \mathrm{~km}$ of side length, discretized by $100 \times 100$ hexahedral elements. In the vertical direction, the basin is formed by 20 stratigraphic units, resulting in a total of 200,000 elements.

One relevant contribution that a more comprehensive geomechanics approach can provide is related to natural fracturing in the basin. A preliminary criterion to predict the possibility of

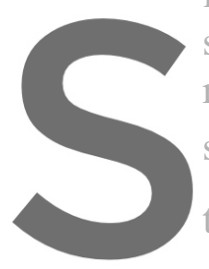
shear-induced fractures right side (compaction) surface [13]. In the present simulation, the e the amount of shear deformation occurred in is the deviatoric part of the plastic strain rate:

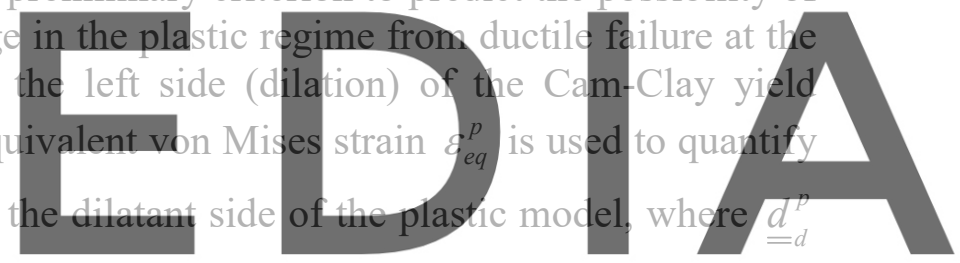

Register for free at https//www.scipedia.comt to download the version without the watermark $\varepsilon_{e q}^{p}=\int_{0} \sqrt{\frac{2}{3}}=d^{p}: d_{d}^{p} \mathrm{~d} t$

A quarter of the model is considered in Fig. 4 to illustrate the present day distribution of the cumulated von Mises strain during shear-induced dilation. The vertical planes represented in the figure intersect each other at the center of the basin, where it is possible to note the uplifted zone and associated erosion. The results indicate two types of failure: 1) totally drained at the surface, 2) undrained at the folding of the lower seal layers. It is important to mention that conventional basin codes are unable to represent such phenomena, which may significantly impact the poromechanical evolution and migration paths of the basin. 


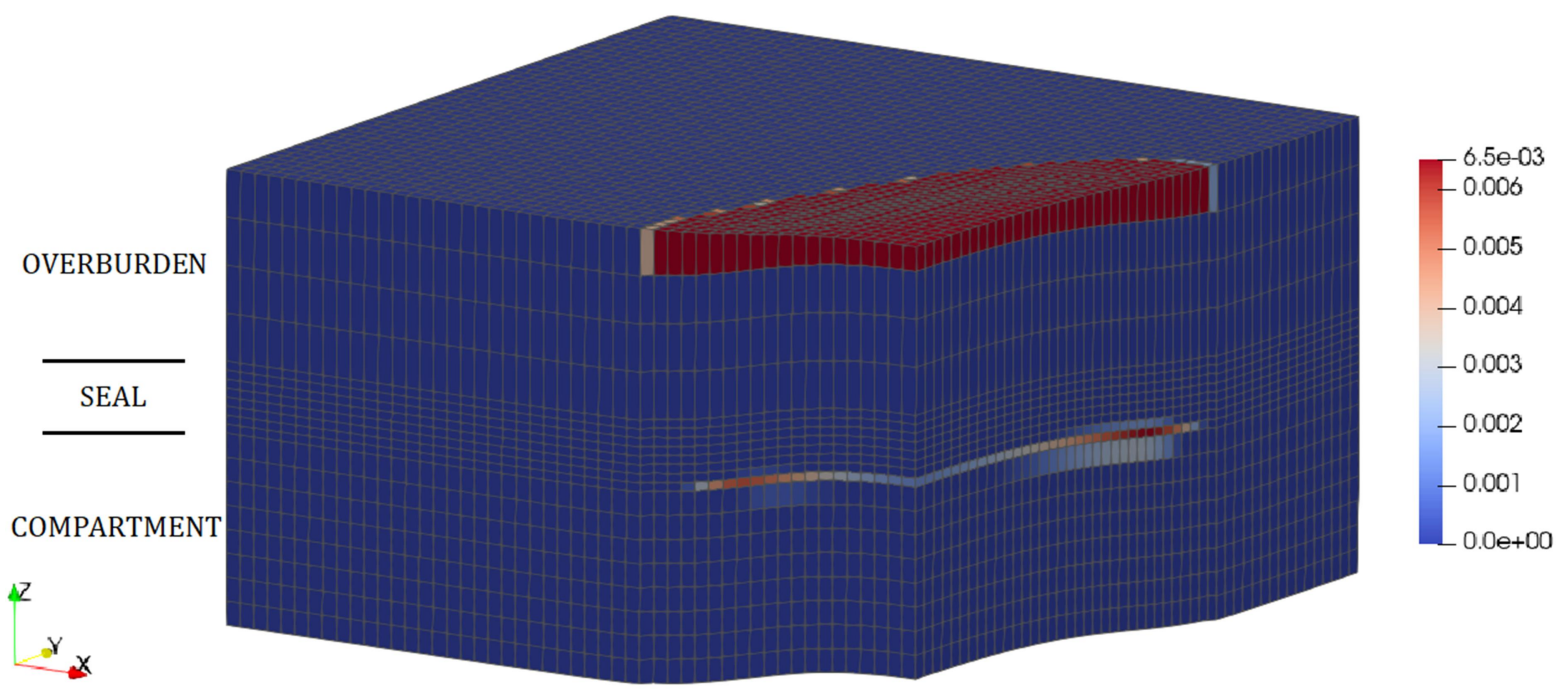

Figure 4: Equivalent shear strain at present day (vertical exaggeration: $3 \mathrm{x}$ )

The stress path in the $\left(-p^{\prime} ; q\right)$ plan can grant critical information to better understand the evolution of geomaterials, where $q=\sqrt{(3 / 2) \underline{\underline{S}}: \underline{\underline{S}}}$ is the equivalent deviatoric stress. It is drawn in Fig. 5 for the cell presenting the highest equivalent shear strain in the folding of the lower seal layer of Fig. 4. The present day Cam-Clay plastic surface $(\mathrm{f}=0)$ is also represented.

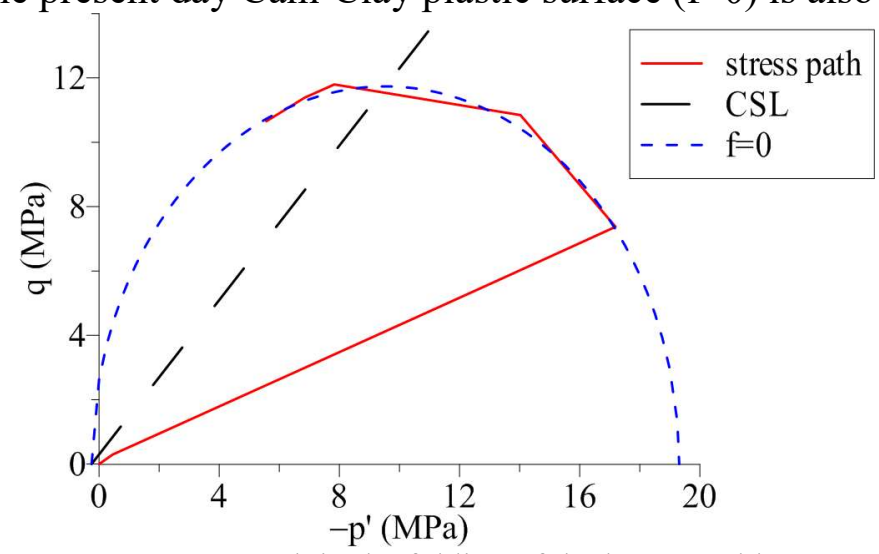

Figure 5: Stress path in the folding of the lower seal layer

From the moment of its deposition up to the beginning of the tectonic event, the stress path follows a straight line defining the burial trend of the material. As the tectonic sequence starts, the stress path direction significantly changes, crossing the critical state line (CSL) and leading to shear-induced dilation. This results from the combination of the overpressure development associated to the lateral loading, together with the vertical unloading due to partial erosion of the uplifted zone of the basin. Similar material behavior has been observed by other authors who investigated the influence of tectonics in sedimentary basins [14]. 


\section{CONCLUSIONS}

- $\quad$ The work presented the importance of including 3D geomechanics into sedimentary basin modeling, also giving insights on the limitations of classical PSM codes and under what circumstances they produce reliable results.

- The proposed workflow is capable of providing a more realistic geomechanical history of the basin, with valuable information regarding seal rock fracturing and identification of critically stressed zones.

- $\quad$ Considering the evolution of the hydrocarbon industry and the increasingly complex conditions in which the search for new resources takes place, a more comprehensive geomechanical approach may help to better understand the geological context of new exploration areas.

\section{REFERENCES}

[1] Allen, P.A. and Allen, J.R. Basin analysis: principles and applications. 2nd ed. Blackwell Science Ltd, (2005).

[2] Kim, J., Tchelepi, H.A. and Juanes, R. Stability and convergence of sequential methods for coupled flow and geomechanics: drained and undrained splits. Comput. Methods Appl. Mech. Eng. (2011) 200:2094-2116.

[3] Dormieux, L. and Maghous, S. Poroelasticity and poroplasticity at large strains. Oil Gas Sci. Technol. - Revue de l'IFP (1999) 54:773-784.

[4] Hashin, Z. Analysis of composite materials - a survey. J. Appl. Mech. (1983) 50:481-505.

[5] Wood, D.M. Soil behaviour and critical state soil mechanics. Cambridge University Press, (1990).

[6] Schneider, F., Potdevin, J.L., Wolf, S. and Faille, I. Mechanical and chemical compaction model for sedimentary basin simulators. Tectonophysics (1996) 263:307-317.

[7] Bathe, K.J. Finite Element Procedures. Prentice-Hall, (1996).

[8] Crisfield, M.A. Non-linear finite element analysis of solids and structures: essentials. John Wiley \& Sons, (1997).

[9] Mainguy, M. and Longuemare, P. Coupling fluid flow and rock mechanics: formulations of the partial coupling between reservoir and geomechanical simulators. Oil Gas Sci. Technol. - Revue de l'IFP (2002) 57:355-367.

[10] Bernaud, D., Dormieux, L. and Maghous, S. A constitutive and numerical model for mechanical compaction in sedimentary basins. Comput. Geotech. (2006) 33:316-329.

[11] Lemos, P.S.B., Brüch, A. and Maghous, S. Coupled elastoplastic-viscoplastic solutions for compaction process in sedimentary basins. In Proceedings of the XL Ibero-LatinAmerican Cong. Comp. Meth. Eng. (2019).

[12] Brüch, A., Maghous, S., Ribeiro, F.L.B. and Dormieux, L. A thermo-poro-mechanical constitutive and numerical model for deformation in sedimentary basins. J. Pet. Sci. Eng. (2018) 160:313-326.

[13] Bemer, E., Vincké, O. and Longuemare, P. Geomechanical log deduced from porosity and mineralogical content. Oil Gas Sci. Technol. - Revue de l'IFP (2004) 59:405-426.

[14] Maghous, S., Brüch, A., Bernaud, D., Dormieux, L. and Braun, A.L. Two-dimensional finite element analysis of gravitational and lateral-driven deformation in sedimentary basins. Int. J. Numer. Anal. Meth. Geomech. (2014) 38:725-746. 